\title{
Electron emission modulation effects in micro-size structures
}

\author{
H. Hartnagel \\ Institut für Hochfrequenztechnik, Technische Universität Darmstadt, Merckstr.25, Darmstadt 64283, Germany \\ E-mail:Hartnagel@hf.tu-darmstadt.de
}

\begin{abstract}
A promising application of field-emission phenomena is microwave high-frequency oscillation generation. In this presentation, new effects at the field emission, as a perspective mechanism of high-frequency oscillation generation, has been investigated and analyzed. The mechanisms are connected with the generation of oscillations in field emission structures (i) based on the silicon or GaAs tips with ultrathin diamond-like carbon (DLC) films, (ii) III-V semiconductors ( $\mathrm{GaAs}, \mathrm{GaN})$ and (iii) $\mathrm{SiGe}$ materials.
\end{abstract}

Keywords: field emission, microwave generation.

Paper received 19.12.02; accepted for publication 18.03.03.

\section{Introduction}

Electron field emission from different materials and structures is intensively investigated due to possibility to use this phenomenon in vacuum microelectronic devices and flat panel field emission displays [1-4]. It is necessary to obtain field-emission cathodes or tip arrays with improved parameters, namely enhanced field-emission efficiency (higher emission current density at lower applied voltage), stability and uniformity of field emission (FE).

As a rule, FE current-voltage characteristics correspond to the Fowler-Nordheim law (straight line in $\lg \left[\mathrm{I} / \mathrm{V}^{2}\right]$ vs. $1 / \mathrm{V}$ coordinates). But for investigations of electron FE from some types of materials and structures, the peculiarities such as oscillations, region with negative differential conductivity, etc. are observed [5-11]. The existence of a region with negative differential conductivity points to the possibilities of using such emitters for oscillation generation. Due to the transport of carriers in vacuum, the frequency of oscillations can be very high (THz).

In this paper we performed the theoretical analysis of electron transport in FE structures, which have I-V characteristics with a negative differential-conductivity region. Some types of cathodes, on the basis of silicon, gal- lium arsenide and $\mathrm{Si} / \mathrm{SiGe}$, were prepared to realize $\mathrm{FE}$ I-V characteristics with negative differential conductivity and ultra-high frequency generation.

\section{Experiment}

The measurements of the emission current from samples were performed in a vacuum system which could be pumped to the stable pressure of $10^{-6}-10^{-7}$ Torr. The emission current was measured in the ungated cathode-anode diode structure.

The emitter-anode spacing $\mathrm{L}$ was constant in the range of 7.5-20 $\mathrm{mm}$. We fabricated a test- diode construction by sandwiching the anode and cathode plates. Silicon or gallium arsenide wafers were used as the cathode and a molybdenum wire or silicon wafer was used as an anode. A 7.5-20 mm-high fluoroplast film spacer was used to keep the two plates separated from each other. The emission current-voltage characteristics were obtained for a current sensitivity of $5 \mathrm{nA}$ over a voltage range up to $1500 \mathrm{~V}$. A $0.56 \mathrm{MW}$ resistor was placed in series with the cathode to provide short-circuit protection.

The silicon tip array $\left(8 \times 8 \mathrm{~cm}^{2}\right)$ was completely formed and the $1 \mathrm{~cm}^{2}$ squares were cut from the wafer and mounted into the vacuum station. The control silicon emitter tip 


\section{H. Hartnagel: Electron emission modulation effects in ...}

array (without coating) was dipped in a 5\% HF solution for $20 \mathrm{~s}$ to remove the native oxide layer immediately before mounting in the HV system. The investigated cathode area for one measurement was $5.5 \times 10^{-3} \mathrm{~cm}^{2}$ and it contained $1.4 \times 10^{3}$ tips.

\section{Results and discussion}

\subsection{Emission from silicon and emitter coated with ultrathin DLC films}

The total field emission (FE) current from uncoated and coated DLC film Si cathodes was calculated as a sum of resonant $J_{r}$ and nonresonant $J_{n r}$ FE current components [9]

$J_{r, n r}=4 \pi q M m^{*} k T / h^{3} \int D_{r, n r} \times \ln \left[1+\exp \left(\left(\varepsilon_{f}-\varepsilon\right) / k T\right)\right] \times d \varepsilon$

where $M$ and $m^{*}$ - the number of equivalent valleys and the longital electron mass in the Si conductive band; $D_{r}\left(\varepsilon, \varepsilon_{r}, F_{c}, \phi\right), D_{n r}\left(\varepsilon, F_{c}, \phi\right)$ - the coefficient of electron resonant and nonresonant transmission through the potential barrier of a cathode; $\varepsilon_{\phi}, \varepsilon_{r}$ and $\varepsilon$-the emitter Fermi energy, the resonant level and tunneling electron energy; $\phi-$ Si-DLC film barrier; $F_{c}$ - electric field in DLC coating. The other designations are generally accepted.
Research of the FE was performed for $5 \times 10^{15} \mathrm{~cm}^{-3}$ emitter doping level, $d=1 \div 30 \mathrm{~nm}$ is the thickness of undoped DLC film, $\phi=0.5 \div 3 \mathrm{eV}$ and $F=5 \times 10^{6} \div 6 \times 10^{7} \mathrm{~V} / \mathrm{cm}$ electrical field in the vacuum range. It was assumed sequential tunneling of electrons through both barriers (DLC film and vacuum, see Fig. 1) in the absence of electrons scattering and charge accumulation in the potential well between barriers. Solid and dash current-field plots on Fig. 2 refer to calculated FE total current and its nonresonant component dependences on the field. Analysis of them have shown that the more $d$ and the less $\phi$, the more sharply the FE current density and its dependence from electric field increase at the two-barrier tunneling, the threshold field of one-barrier tunneling and a current-field dependence at this mode decrease. Permeability of the cathode potential profile at two-barrier tunneling is determined by the degree of a triangular potential well bottom fall. And the thicker DLC film and the smaller the barrier $\phi$, the lower the well bottom falls at the given field value. Therefore at rather large $d(8 \mathrm{~nm})$ and small $\phi(0.5 \mathrm{eV})$ the FE current increases as much as $10^{8}$ times at fields increasing from $10^{7}$ to $2 \times 10^{7} \mathrm{~V} / \mathrm{cm}$ and it is almost saturated at the threshold field of the onebarrier tunneling mode. The saturation current density is identical at different $d$ and increases for a $\phi$ reduction. Increasing $\mathrm{d}$ more than optimum (near $10 \mathrm{~nm}$ ) leads to a lowering of the FE minimum electric field but not of the field corresponding to the maximum FE current. With a
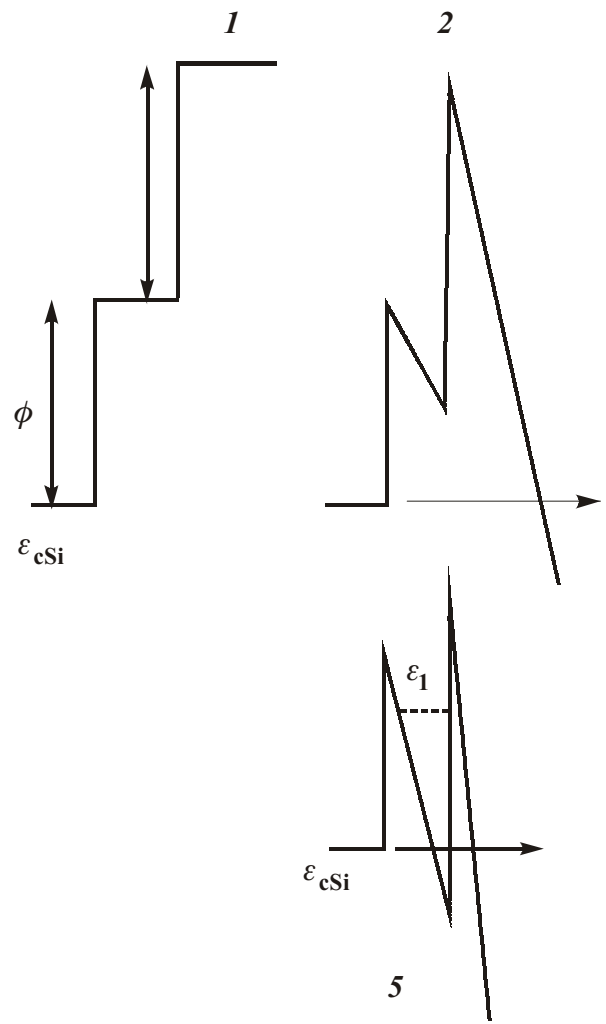

4

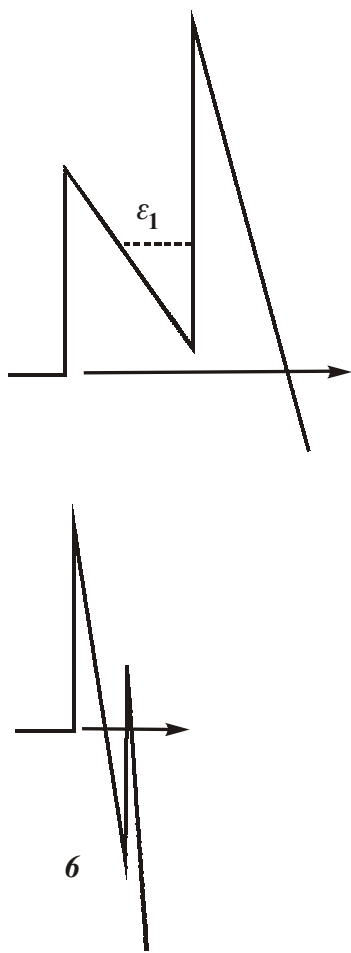

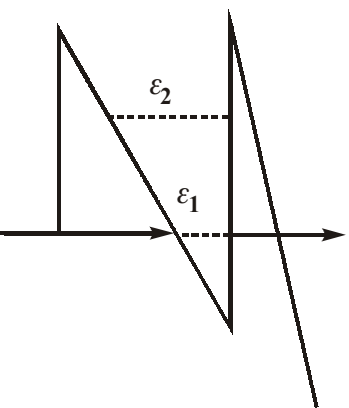

Fig. 1. Energy band diagrams of Si-DLC film cathodes: $I$ - without applied field; $2-6$ - with applied field. 


\section{H. Hartnagel: Electron emission modulation effects in ...}

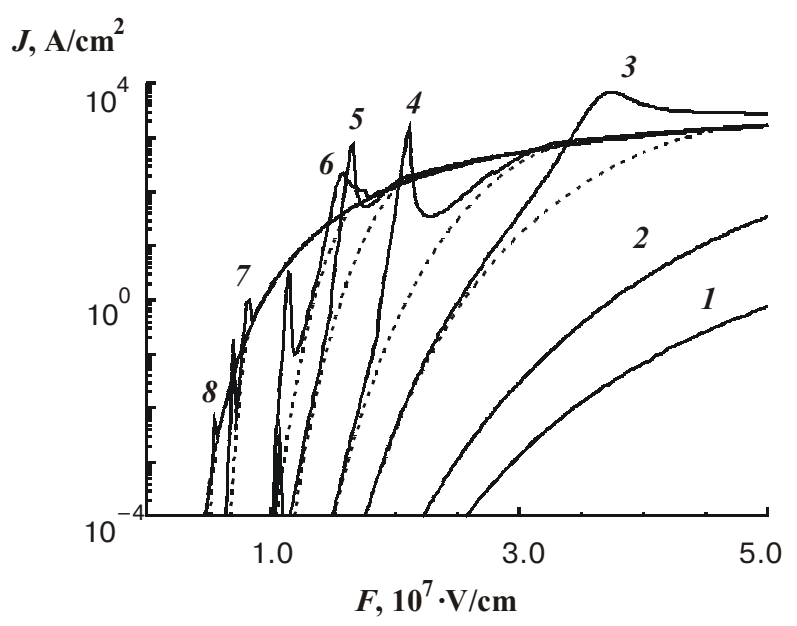

Fig. 2. FE current-field plots for uncoated $(1)$ and coated $(2-8)$ cathode with thickness of DLC coating $d=1 ; 3.5 ; 5 ; 8 ; 10 ; 20 ; 30$ $\mathrm{nm}$ correspondingly and $\phi=1.3 \mathrm{eV}$.

reduction of $d$ and an increase of $f$ the current-field dependence of the cathode with DLC coating comes nearer to the current-field dependence of the common cathode.

Taking into account electron wave interference in the triangular quantum well between the potential barriers, the processes of resonant FE for the cathode with DLC coating were investigated. It was supposed, that the electron impulse relaxation time constant in the DLC film is about that in the bulk i-GaAs at $300 \mathrm{~K}\left(3 \times 10^{-13} \mathrm{sec}\right)$. The resonant FE processes in the cathode with DLC coating cause resonant current maxima, whose width decreases with film thickness increasing. The maxima shift towards the higher electric field with a reduction of $d$ and an increasing of $\phi$ and vanish at an electric field higher than the threshold field of one-barrier tunneling. The greatest values the total current overall in resonant maximum and the following minimum (in 40 times) and the resonant to nonresonant current ratio $\left(10^{3}\right)$ take place for an optimum $d=5 \mathrm{~nm}$ at $\phi=1.3 \mathrm{eV}$. At a rather small film thickness (the smaller $\phi$, the more is $d$ reduced) resonant tunneling is absent because the resonant levels in a quantum well are absent at rather large electric field.

The results of experimental investigations of electric field emission from DLC film coated Si tip arrays are presented in Fig. 3 (a, b). As can be seen, a resonant peak in emission current is observed. The resonant enhancement of current density is by a factor 2 for tips and 1.7 for flat Si-DLC film cathodes. The of I-V characteristics in Fig. $3(a, b)$ also show the enhancement of the emission current with increasing thickness of the DLC film. This is due to the greater lowering of the output barrier for thicker DLC films. We observed that, for samples with an identical phase structure of the DLC film, the field enhancement parameter $b$ increases with increasing thickness of the DLC film. On the other hand, for thicker DLC films on these tip cathodes, the threshold voltage and resonant peak position shift to smaller voltages (see Fig. 3 (a) curves $1,2)$. The data on electron field emission from GaAs tips coated by ultrathin (10nm) DLC film are shown in Fig. 4.

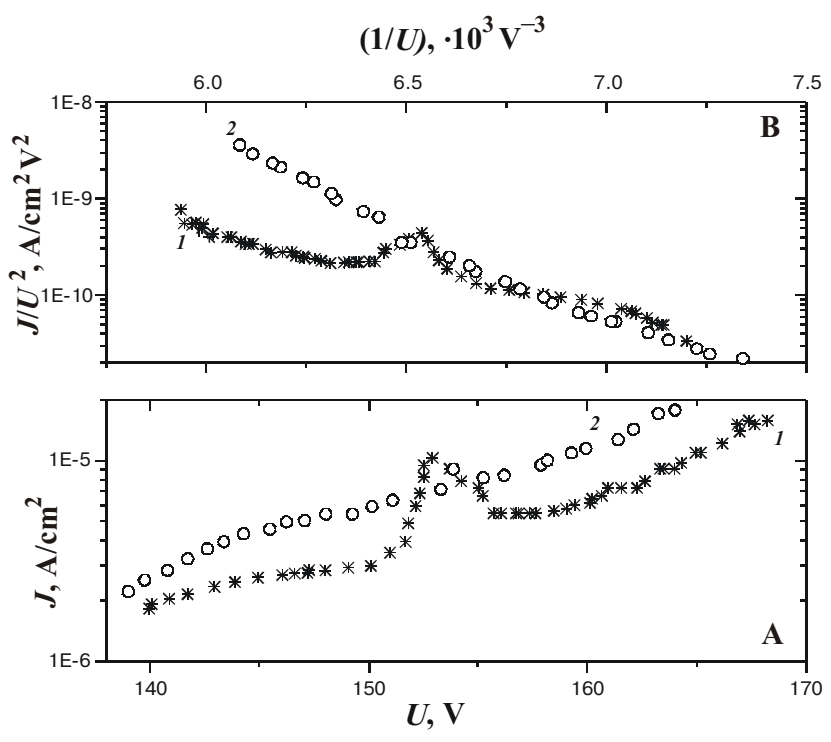

Fig. 3. Experimental current density on voltage dependences (A) and corresponding Fowler-Nordheim plots (B) for silicon tips cathodes with DLC films: $1-d_{\text {DLC }}=8 \mathrm{~nm} ; 2-d_{\mathrm{DLC}}=30 \mathrm{~nm}$.

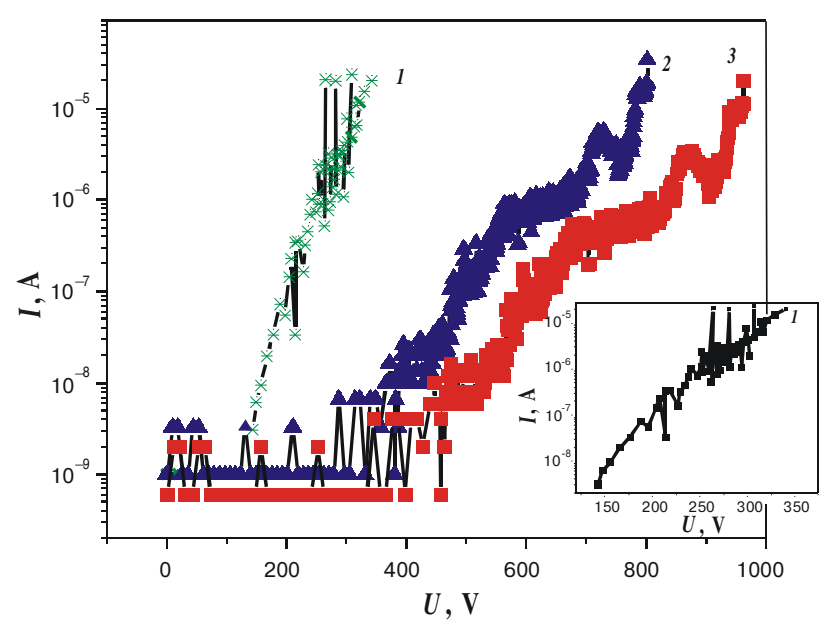

Fig. 4. Current-voltage characteristics of electron field emission from GaAs tips: 1 - without DLC coating; 2,3 with DLC $(d=$ $=10 \mathrm{~nm})$ coating $(3$ - repeated measurement, in insert - curve $I$ in other scale).

\subsection{Peculiarities of FE from GaAs cathodes}

A method for direct generation of a bunched electron beam from a cathode by using GaAs Gunn emitters has been proposed and experimentally verified $[6,10,11]$. This method is based on the Gunn effect [12]. The frequency of the bunched electron beam is approximately equal to the reciprocal of the carrier transit time across the length of the sample, which is the same as in the conventional Gunn diodes, and the operation frequency covers microwave and millimetre wave regions. 


\section{H. Hartnagel: Electron emission modulation effects in ...}

Lateral and vertical configurations of the field-emitter structure have been considered. The pictures of the GaAs lateral field emitter, and of the GaAs vertical field emitter with an air bridge and a cantilever anodes, are shown in Fig. 5. Lateral field-emission devices present advantages of simple fabrication, precise control of electron distance, and lower capacitance. A direct and accurate control of the electrode distance can be achieved via photolithography [6]. Two different doping concentrations have been used for the $2 \mu \mathrm{m}$ thick $n-G a A s$ active layer. The concentration of $1 \times 10^{15} \mathrm{~cm}^{-3}$ and $1 \times 10^{18} \mathrm{~cm}^{-3}$ have been chosen to allow a comparison of emission characteristics. The first satisfies the condition for the formation of a travelling dipole in the emitter. For an emitter length of $70 \mu \mathrm{m}$, the expected frequency of the bunched beam is about $1.3 \mathrm{GHz}$. Fig. 6 shows a current-voltage characteristic for the emitter with the doping concentration of $1 \times 10^{15} \mathrm{~cm}^{-3}$. Electron emission occurs at about $60 \mathrm{~V}$. This electron emission is considered to be a field emission from the slope of the straight line in the FowlerNordheim plot. However, the emission current reaches a maximum value at about $110 \mathrm{~V}$ and then becomes unstable. This current-voltage characteristic is quite similar to that of a bulk GaAs diode with a Gunn oscillation, except for the threshold voltage of around $60 \mathrm{~V}$. In conventional Gunn diodes, such a threshold voltage does not appear. For the emitter with a doping concentration of $1 \times 10^{18} \mathrm{~cm}^{-3}$, the emission current showed a monotonic increase with increasing bias voltage. As expected for the emitter with a heavy doping level, the Gunn oscillation does not occur. These results suggest that the emission above $110 \mathrm{~V}$ in the first emitter is the field emission of accumulated electrons in the travelling dipole domains formed in the emitter.
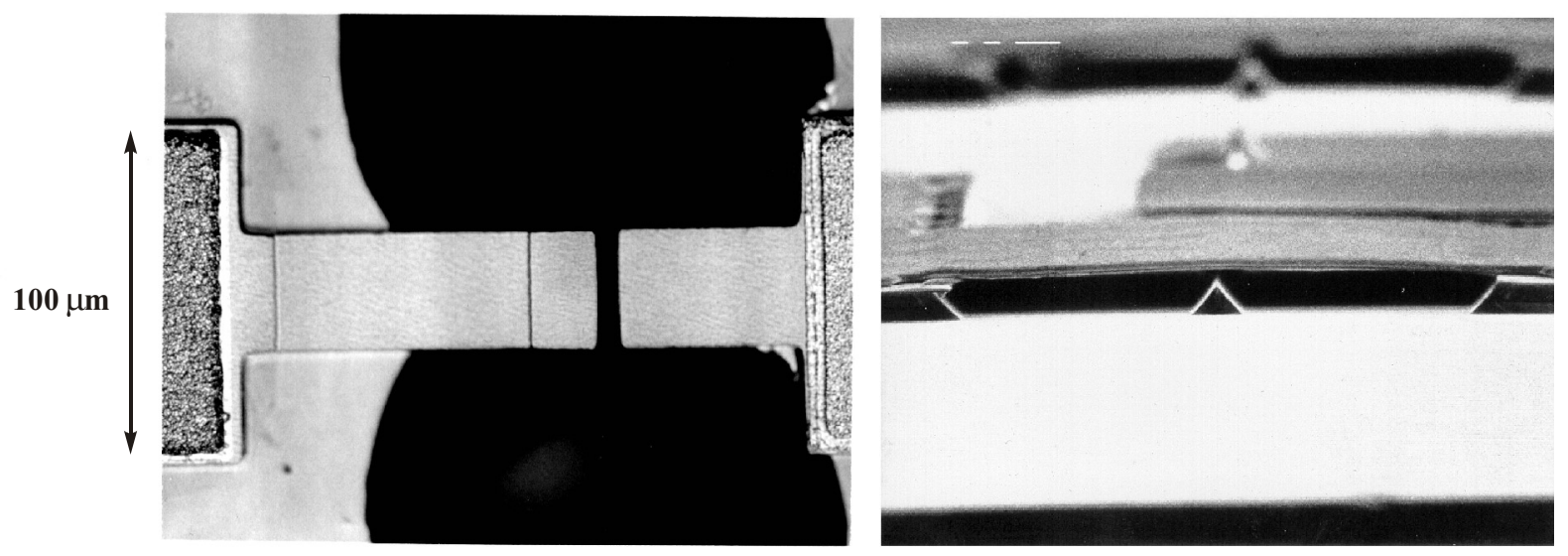

Fig. 5. Picture of the GaAs lateral field emitter and the GaAs vertical field emitter with an air bridge and cantilever anodes.
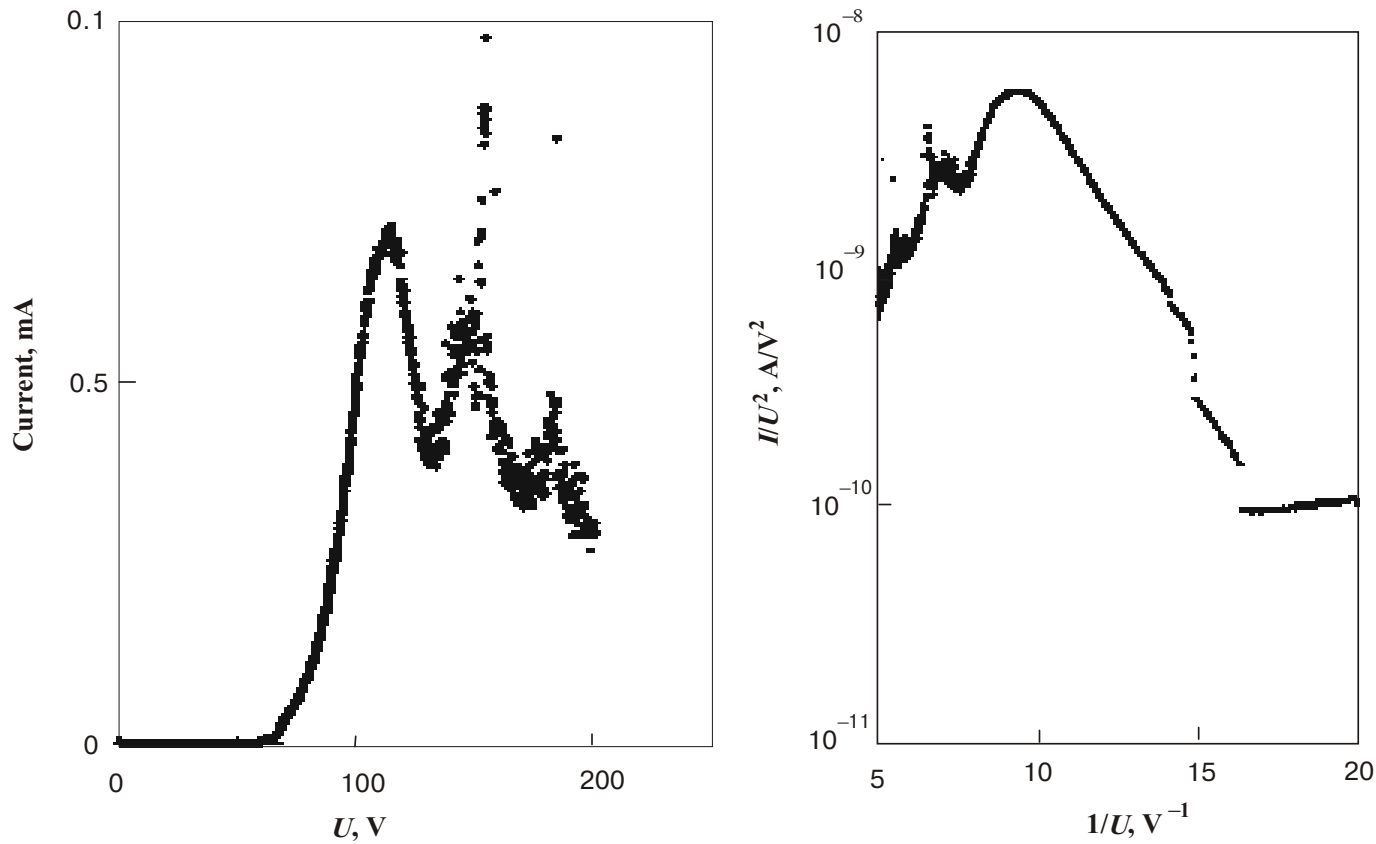

Fig. 6. Current-voltage characteristic $(a)$ and the corresponding Fowler-Nordheim plot $(b)$ of the lateral GaAs Gunn effect field emitter. 


\section{H. Hartnagel: Electron emission modulation effects in ...}

The vertical field emitters were formed on a $n$-GaAs substrate with $1 \times 10^{18} \mathrm{~cm}^{-3}$ doping concentration. The emitter height of about $9 \mu \mathrm{m}$ could be reached. Gold air bridge-emitter distances between $1 \mathrm{~mm}$ and $3 \mathrm{~mm}$ was realized. An emission current without oscillations was observed between the gold air bridge and the emitter for a bias voltage below $14 \mathrm{~V}$. Further work on the development of a vertical field emitter with $1 \times 10^{15} \mathrm{~cm}^{-3}$ doping concentration is done, in order to achieve the travelling dipole domains.

\subsection{Field emission from GaN films}

The quantum dots were formed on the surfase of a GaN layer. The GaN layers were grown by low-pressure metalorganic chemical-vapor deposition (MO CVD) on (0001) c-plane sapphire. The quantum-dot formation was performed by photoelectrochemical (PEC) etching [13] of GaN only under very specific process conditions that produces a high etch selectivity at the dislocations. The GaN layer with quantum dots shown in Fig. 7a was etched for $6 \mathrm{~min}$ in a stirred $0.1 \mathrm{M} \mathrm{KOH}$ solution with a photocurrent of $3 \mathrm{~mA}$. The investigated structure produces a stable current density of $J=40 \mathrm{~mA} / \mathrm{cm}^{2}$ in the high field region. The threshold voltage is $120 \mathrm{~V}$ for this sample [14].

We suggest that the decreased effective work function and hence the turn-on voltage is a result of two factors: the tip radii with increased field enhancement factor and the appearance of a quantum-size effect. The latter causes the splitting of electron energy levels. The characteristic oscillations of the emission current have been observed with increasing bias voltage (Fig. 7b). This is attributed to emission-charging effects occurring in tips with quantum-dot size. The latter contain a small number of electrons and are charged after an emission cycle. The oscillations are obtained for the low field region where the lowering of the work function due to the quantization of electron states in the quantum dot is more pronounced.
With the field growing further the barrier for field emission becomes practically transparent, and almost all the electrons are tunneling through the barrier regardless of their distribution pattern. The effect of quantum size becomes negligible.

\subsection{Field emission from Si/SiGe quantum well structures}

The $\mathrm{Si} / \mathrm{SiGe}$ structures are intensively investigation for different applications $[15,16]$. The fabrication of high speed electronic devices requires abrupt interfaces and flat layers. However, strained $\mathrm{Si}_{1-\mathrm{x}} \mathrm{Ge}_{\mathrm{x}}$ layers deposited on Si generally present highly corrugated surfaces. This feature of surface can be used for electric field enhancement at electron field emission. Changing the $\mathrm{Ge}(\mathrm{x})$ content in $\mathrm{Si}_{1-\mathrm{x}} \mathrm{Ge}_{\mathrm{x}}$ layers, we can rule the parameters of a quantum well. In some cases, the multiplayer $\mathrm{Si}_{1-\mathrm{x}} \mathrm{Ge}_{\mathrm{x}}$ structures are produced to minimize the strain and obtain desirable $\mathrm{Si}_{1-\mathrm{x}} \mathrm{Ge}_{\mathrm{x}}$ quantum well parameters. In our experiment the electron field emission from $\mathrm{Si} / \mathrm{SiGe}$ structures with $\mathrm{SiGe}$ layer grown by molecular beam epitaxy has been investigated. The current-voltage characteristic of electron field emission in a Fowler-Nordheim plot is shown in Fig. 8. Two region with negative differential conductivity are observed. The appearance of peaks is caused by a resonance tunneling effect in structures with a quantum well. There are separate energy levels in SiGe quantum wells between Si and vacuum. At definite electric fields the increase of emission electron current is observed due to including additional mechanism of current transport, i.e. resonance tunneling.

\section{Conclusion}

The peculiarities of electron field emission into vacuum from different quantum size structures have been investigated. Among the structures studied were silicon and

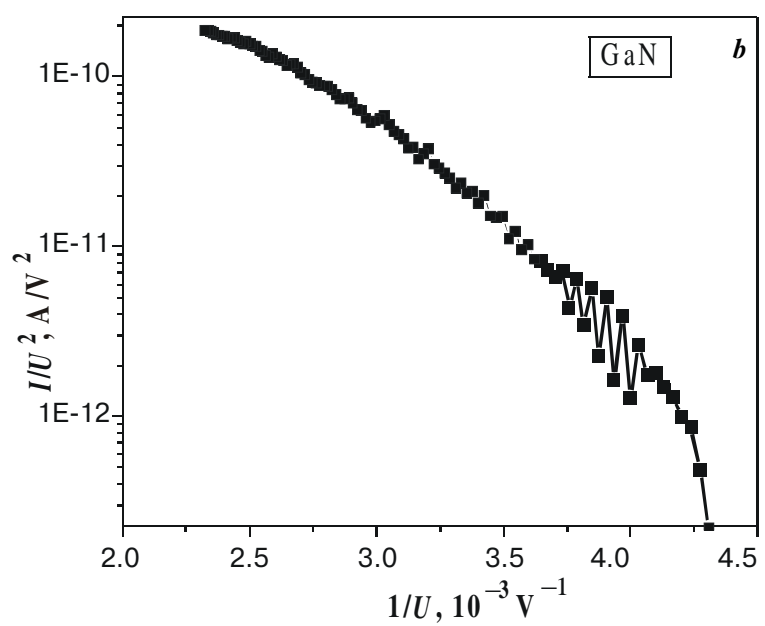

Fig. 7. SEM micrograph of GaN surface $(a)$ and Fowler-Nordheim plot of electron field emission current from GaN layer $(b)$. 


\section{H. Hartnagel: Electron emission modulation effects in ...}

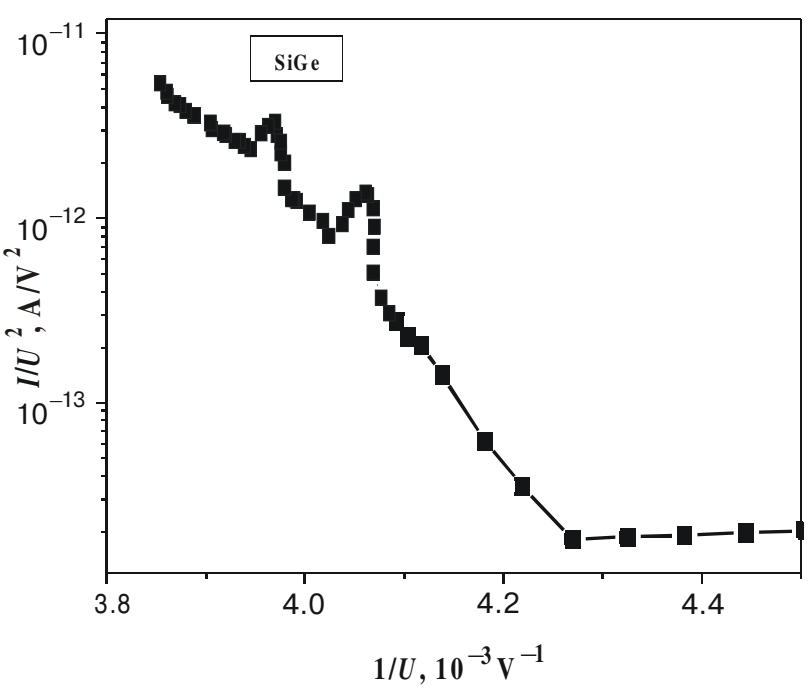

Fig. 8. Fowler-Nordheim plot of electron field emission current from $\mathrm{Si} / \mathrm{SiGe}$ structure with quantum well.

GaAs tips coated with an ultrathin DLC layer, GaAs cathodes, GaN layers and $\mathrm{Si} / \mathrm{SiGe}$ heterostructures. The characteristic peculiarities of electron field emission from these structures appeared of a region with negative deferential conductivity on the current-voltage characteristics of electron field emission. Such structures are promising for high frequency oscillation generation. The models of electron fields emission from structures with quantum well based on resonance tunneling phenomena have been proposed.

\section{Acknowledgments}

This work was supported in part by the DLR (BMBF) in connection with a German-Ukrainian project (UKR 01/ 053) entitled "Electron Emission Properties of the DLC Films and C-Nanotubes" and in part by the "Deutsche Forschungsgemeinschaft (DFG)" in connection with a special research project (SFB 241) on innovative mechatronics.

\section{References}

1. I. Brodie, C.A. Spindt, Adv. In: Electronics and Electron Phys. 83 (1996) 1.

2. R.T. Fulks, G.N. Parsons, D.E. Slobodin, T.N. Yuzuriha. Flat Panel Display Materials III, MRS Sympos. Proc. V.471, Pittsburg, Pensylvania, 1997.

3. S. Lee, Su. Lee, Sun. Lee, D. Jeon. J. Vac. Sci. Technol. B 15 (1991) 457.

4. J.H. Jung, B.K. Ju, H. Kim, Y.H. Lee, S.J. Chung, M.H. Oh. IEEE Trans. Electron. Dev. 45 (1998) 2232.

5. A.A Evtukh, E.B. Kaganovich, V.G. Litovchenko, Y.M Litvin, D.V. Fedin, E.G. Manoilov, S.V. Svechnikov, Mat. Sci.\& Engin. C 19 (2002) 401.

6. Yilmazoglu O., Hartnagel H., Mimura H., Okamura K., Shimawaki H., Yokoo K., In: Proc. $2^{\text {nd }}$ European Field Emission Workshop (Segonia, Spain) 2000.

7. A.I. Kosarev, A.N. Andronov, A.J. Vinogradov, T.E. Felter, A.N. Titkov, I.V. Makarenko, Z. Vaqar, S.V. Robozerov, M.V. Shutov. Proc. 12 th Int. Vac. Microelectron. Conf. (6-9 July, Darmstadt, Germany) 1999, 418.

8. K. Okano, T. Yamada, A. Sawabe, S, Koizumi, J. Itoh. Proc. $12^{\text {th }}$ Int. Vac. Microelectron. Conf. (6-9 July, Darmstadt, Germany) 1999, 420.

9. A. Evtukh, H. Hartnagel, V. Litovchenko, O. Yilmazoglu. Proceedings IFES 2001, 2001.

10. O. Yilmazoglu, H. Mimura, K. Mutamba, H. Hartnagel, K. Okamura, H. Shimawaki, und K. Yokoo. Proceedings IVEC 2001, Nordwijk, The Netherland, pp. 243-246, 2001.

11. K. Yokoo, K. Okamura, H. Hasegawa, H. Mimura, O. Yilmazoglu, K. Mutamba, H. Hartnagel, H. Ishizuka, und Y. Kawamura. Proceedings of 8th Int. Symp. On Microwave and Optical Technology 2001, Montreal, Canada, pp. 27-30, 2001.

12. Sze S.M. Semiconductor Sensors, John Wiley \& Sons, INC., 1994.

13. C. Youtsey et al.. Appl. Phys. Lett, 74 (23) (1999) 3537.

14. O. Yilmazoglu, D. Pavlidis, Yu. M. Litvin, S. Hubbard, I.M. Tiginyanu, K. Mutamba, H.L. Hartnagel, V.G. Litovchenko, A. Evtukh. Proceedings of IVMC 2002, 2002.

15. D. Grutzmacher, R. Hartmann, P. Schnappauf, U. Gennser, H. Sigg, D. Bachle, S. Brosig. J. Vac. Sci. Technol. B16(3) (1998) 1670 .

16. I. Berbezier, B.Gallas, L.Lapena, J.Fernandez, J. Derrien, B. Joyce. J. Vac. Sci. Technol. B16(3) (1998) 1582. 